\title{
Fabrication and characterization of PEDOT nanowires based on self-assembled peptide nanotube lithography
}

Andersen, Karsten Brandt; Christiansen, Nikolaj Ormstrup; Castillo, Jaime; Rozlosnik, Noemi; Svendsen, Winnie Edith

Published in:

Organic Electronics

Link to article, DOI:

10.1016/j.orgel.2013.02.033

Publication date:

2013

Document Version

Publisher's PDF, also known as Version of record

Link back to DTU Orbit

Citation (APA):

Andersen, K. B., Christiansen, N. O., Castillo, J., Rozlosnik, N., \& Svendsen, W. E. (2013). Fabrication and characterization of PEDOT nanowires based on self-assembled peptide nanotube lithography. Organic Electronics, 14(5), 1370-1375. https://doi.org/10.1016/j.orgel.2013.02.033

\section{General rights}

Copyright and moral rights for the publications made accessible in the public portal are retained by the authors and/or other copyright owners and it is a condition of accessing publications that users recognise and abide by the legal requirements associated with these rights.

- Users may download and print one copy of any publication from the public portal for the purpose of private study or research.

- You may not further distribute the material or use it for any profit-making activity or commercial gain

- You may freely distribute the URL identifying the publication in the public portal 


\title{
Fabrication and characterization of PEDOT nanowires based on self-assembled peptide nanotube lithography
}

\author{
Karsten Brandt Andersen ${ }^{1}$, Nikolaj Ormstrup Christiansen ${ }^{1}$, Jaime Castillo-León *, \\ Noemi Rozlosnik, Winnie Edith Svendsen
}

DTU Nanotech, Department of Micro- and Nanotechnology, Technical University of Denmark, Lyngby, Denmark

\section{A R T I C L E I N F O}

\section{Article history:}

Received 14 December 2012

Received in revised form 6 February 2013

Accepted 23 February 2013

Available online 13 March 2013

\section{Keywords:}

PEDOT:TsO

Diphenylalanine

Nanowire

Nanolithography

Cleanroom fabrication

Self-assembly

\begin{abstract}
A B S T R A C T
In this article we demonstrate the use of self-assembled peptide nanotube structures as masking material in a rapid, mild and low cost fabrication of polymerized p-toluenesulfonate doped poly(3,4-ethylenedioxythiophene) (PEDOT:TsO) nanowire device. In this new fabrication approach the PEDOT:TsO nanowire avoids all contact with any organic solvents otherwise traditionally used in clean room fabrication. This can be achieved due to the intriguing properties of the self-assembled peptide nanotubes utilized as a dry etching mask for the patterning of the PEDOT:TsO nanowire. The peptide nanotubes, despite remaining stable during the reactive ion etching procedure, can be dissolved rapidly in water afterwards. The fabricated PEDOT:TsO nanowire devices exhibit excellent electrical characteristics. Finally, the potential of PEDOT:TsO nanowires as temperature sensors has been demonstrated and the high resolution of the sensor was illustrated.
\end{abstract}

(c) 2013 Elsevier B.V. All rights reserved.

\section{Introduction}

Since the original article on silicon nanowire demonstrating the potential of these new types of biosensors was published more than a decade ago [1] the field of silicon nanowires has received a lot of attention and many articles exploring this specific field have been published [2-5]. However, the fabrication costs of the nanowire devices still are a challenge hindering the full exploration of the nanowire devices as biosensors for diagnostic use [6]. One of the approaches explored to face this challenge has been the fabrication of nanowires in cheaper materials, for instance conductive polymers such as polyanaline [79] and poly(3,4-ethylenedioxythiophene) (PEDOT) [1012]. The benefits of the cheaper nanowire devices have been demonstrated in various applications ranging from chemical gas and liquid sensors $[13,14]$, over temperature sensors [15] to biosensors [16-18]. However, the fabrica-

\footnotetext{
* Corresponding author. Tel.: +45 768521038 .

E-mail address: Jaime.castillo@nanotech.dtu.dk (J. Castillo-León).

1 These authors contributed equally to this work.
}

tion procedure for the polymer nanowire devices still is challenging and/or time consuming due to the incompatibility of the polymers with organic solvents used in traditional nanofabrication techniques [19]. Recently, we have demonstrated the use of self-assembled diphenylalanine peptide nanotubes (PNT's) as dry etching masks for the low cost, mild and rapid clean room fabrication of silicon nanowire devices [20,21]. Diphenylalanine self-assembled peptide nanostructures are biological entities able to selforganize in a rapid way under mild conditions. Its on-chip fabrication, structural and electrical characterization, manipulation and application in the development of biosensors were recently reported [22-26]. In this work we have demonstrated the rapid fabrication of polymerized p-toluenesulfonate doped poly(3,4-ethylenedioxythiophene) (PEDOT:TsO) nanowire devices (the whole process can be conducted in approximately $5 \mathrm{~h}$ ) based on the self-assembled PNT lithography. The benefit of this fabrication procedure is that any contact between PEDOT:TsO and organic solvents, such as acetone, can be avoided since the PNT's dissolve rapidly in Milli Q water after the dry etching pattern transfer [27]. 


\section{Materials and methods}

\subsection{Chemicals}

The lyophilized diphenylalanine dipeptide powder was purchased from Bachem (product number: G-2925). All other chemicals utilized were purchased from SigmaAldrich.

\subsection{PEDOT:TsO preparation}

$260 \mu \mathrm{l}$ Baytron C (40\% Fe $\mathrm{Fe}^{\mathrm{III}}$ tosylate in butanol), $80 \mu \mathrm{l}$ butanol, $6 \mu$ l pyridine and $8.8 \mu$ l EDOT were thoroughly mixed and spun on the substrate wafer with $4000 \mathrm{rpm}$ for $60 \mathrm{~s}$. The coated wafers were heated to $70^{\circ} \mathrm{C}$ for $10 \mathrm{~min}$ to evaporate the inhibitor Pyradine and start the polymerization process. The wafers were finally rinsed in de-ionized water to wash away excess reactants. This procedure ensures a PEDOT:TsO film thickness of $75 \mathrm{~nm}$.

\subsection{Preparation of diphenylalanine peptide nanotubes}

The PNT's were prepared from a stock solution, where the lyophilized form of the peptide monomers was dissolved at a concentration of $100 \mathrm{mg} / \mathrm{ml}$ in 1,1,1,3,3,3-hexafluoro-2-propanol (HFP). The HFP stock solution was diluted to a final peptide concentration of $2 \mathrm{mg} / \mathrm{ml}$ in water in which process the PNT's are formed. Fresh stock solution was prepared prior to experiments to avoid pre-aggregates.

\subsection{Spin casting procedure}

The PNT's were positioned across the electrodes in a spin casting procedure as described in [20]. In short, the solution containing the newly formed PNT's is dropped in individual drops on the spinning substrate. This spin casting procedure ensures that the PNT's on the surface of the wafer are oriented along the axial direction. In this work a spin rate of $4000 \mathrm{rpm}$ was utilized in all the experiments.

\subsection{Reactive Ion etching procedure}

The reactive ion etching procedure was conducted in a STS Cluster System C010 with a pressure of 300 mTorr and a power of $100 \mathrm{~W}$. For the patterning of the PEDOT:TsO an oxygen based plasma was used (98 SCCM O 2 and 20 SCCM $\mathrm{N}_{2}$ ). The wafers were subjected to the plasma for $15 \mathrm{~s}$, which was enough to etch through the thin PEDOT:TsO layer.

\subsection{Visualization}

All scanning electron microscopic (SEM) images were acquired using a Zeiss SUPRA ${ }^{\circledR} 40$ VP operated in the inlens mode with an acceleration voltage of $3 \mathrm{kV}$. The atomic force microscopy (AFM) measurements were conducted using a PSIA XE 150 in both tapping (for topography imaging) and contact (for conductive recordings with a tip bias of $0.7 \mathrm{~V}$ ) mode. The conductive AFM images were acquired with a $\mathrm{Cr} / \mathrm{Pt}$ coated cantilever (ContE-Al, Budget Sensors) with a force constant of $0.3 \mathrm{~N} / \mathrm{m}$. The current between the AFM tip and sample was measured using an inverting current amplifier and one of the analog-digital converter inputs of the AFM controller.

\subsection{Electrical readout}

The impedance of the PEDOT:TsO nanowire device was recorded with a custom build lab view controlled experimental set-up, as described in [3]. In this set-up the current through the PEDOT:TsO nanowire is externally amplified using a low-noise current preamplifier model: SR570 from Stanford Research Systems and finally recorded using a National Instruments data acquisition card model BNC-2111.

\section{Results and discussion}

\subsection{Fabrication of PEDOT:TsO nanowires}

In a previous work, we have demonstrated the use of the diphenylalanine PNT's as a dry etching mask for the fabrication of poly silicon nanowires [20,21]. One of the benefits of utilizing the PNT's besides the low cost and rapid fabrication process is that they can be removed in Milli Q water after processing [27]. In this way, acetone traditionally required for the removal of photoresists, can be avoided. This enables the patterning of new types of materials normally incompatible with such organic solvents. In this work, a modified version of this fabrication procedure has been utilized for the rapid and low cost fabrication of PEDOT:TsO nanowire devices. In fact the entire fabrication procedure can be conducted in approximately $5 \mathrm{~h}$ yielding at the moment around 200 ready to use PEDOT:TsO nanowire devices. In Fig. 1, the fabrication steps in the process are illustrated. The first and most time consuming part of the fabrication was the deposition and patterning of the gold electrodes and contact pads allowing electrical connection to the PEDOT:TsO nanowires. In this work $90 \mathrm{~nm}$ of gold was deposited on $500 \mathrm{~nm}$ thick silicon dioxide layer grown from a bare silicon wafer utilizing a $10 \mathrm{~nm}$ chromium adhesion layer. The metal layers were patterned in a traditional lift off procedure. The only difference being that the wafer was dipped in a $5 \%$ buffered hydrofluoric acid solution for $20 \mathrm{~s}$ prior to metal deposition. This ensure a smooth corner on the edge of the gold electrodes to provide electrical connection between the electrodes and the PEDOT:TsO nanowire. In the second step, the PEDOT:TsO layer was spin coated on the wafer with a spin rate of $4000 \mathrm{rpm}$ followed by a post backing step as explained above. The PNT's forming the dry etching mask were positioned across the electrodes in a modified spin casting technique described in [20]. In this manipulation procedure the PNT's aligned according to the axial direction on the wafer. The gold electrodes were positioned perpendicular to this direction. In this way the aligned peptide nanotubes were able to bridge the gap at the electrode position and not at other locations in order to avoid potential short circuits as described in our previous work [20]. The final step in the fabrication process was to transfer the pattern of the self-assembled peptide nanotubes to the spin coated 

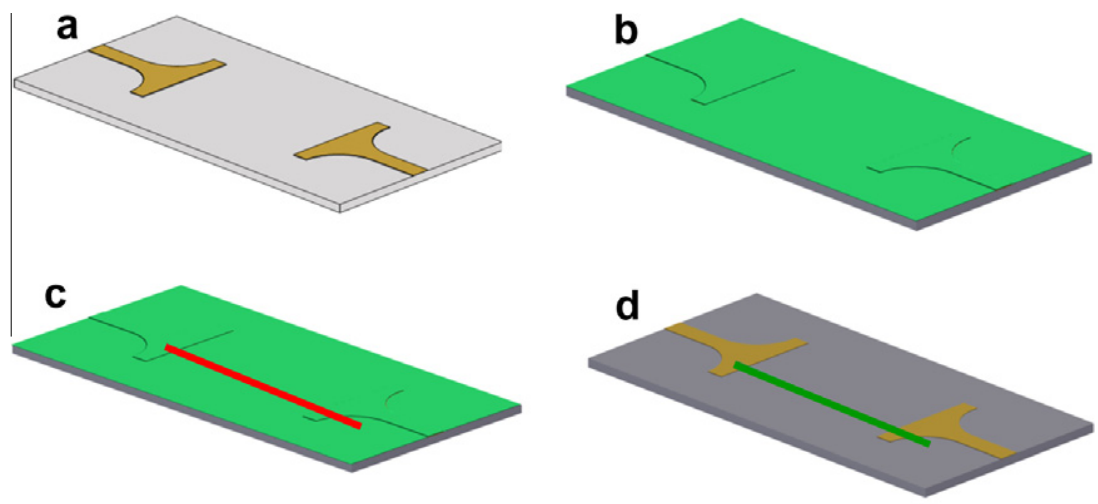

Silicon Substrate

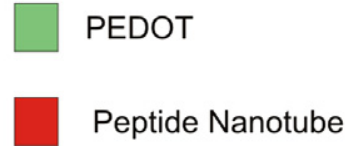

Gold

Peptide Nanotube

Fig. 1. Illustration of the fabrication procedure developed in this work. In this approach the gold electrodes were initially defined by a lift off procedure. The PEDOT:TsO was spin coated on the wafer and finally the peptide nanotubes were spin casted on the coated wafer to ensure proper alignment of the structures to the electrodes. In the last step the pattern of the nanotubes was transferred to the PEDOT:TsO layer in a reactive ion etching procedure and finally the peptide nanotubes were dissolved in Milli Q water. The process can be completed in approximately 5 h.

PEDOT:TsO layer. After pattern transfer the PNT's were removed in pure Milli $\mathrm{Q}$ water.

\subsection{Characterization of PEDOT:TsO nanowires}

The fabricated PEDOT:TsO nanowire devices were characterized using both SEM and AFM. A SEM image of a PEDOT:TsO nanowire spanning the gap between two gold electrodes is shown in Fig. 2a. Fig. 2b displays a zoom of the contact between the PEDOT:TsO nanowire and the gold electrode illustrating the smooth step coverage of the PEDOT:TsO nanowire across the step from the oxide substrate to the top of the gold electrodes. The large contact area between the gold electrodes and the PEDOT:TsO nanowire and the smooth coverage of the step from the electrode to the substrate by the PEDOT:TsO, as seen in the figure, ensured proper electrical contact in the devices. This is also evident from the small impedance and linear current voltage characteristics, even at room temperature, of these devices as described below. In Fig. 2c, a topography image of the surface of the PEDOT:TsO nanowire recorded with an AFM in tapping mode is shown. Based on the line profile shown in Fig. 2d, the height of the PEDOT:TsO nanowire can be determined to be $75 \mathrm{~nm}$. In this image the surface roughness of the PEDOT:TsO nanowire is also clearly seen. The roughness of the surface introduces a larger surface to volume ratio than comparable flat nanowires and should therefore increase the sensitivity of the measurements. Using conductive AFM the complete removal of the PNT's from the PEDOT:TsO surface after etching was verified as seen in Fig. 3b. From this AFM image it is seen that the surface of the PEDOT:TsO nanowire is highly conductive and hence the isolating PNT's must have dissolved.

\subsection{Electrical characterization}

From the SEM images in Fig. 2a and b a continuous connection between the PEDOT:TsO nanowire and the gold surface without any fracture in the structure can be seen, indicating a good electrical connection. To verify this, the current-voltage relationship of a single PEDOT:TsO nanowire was recorded and plotted in Fig. 3a for a range of different temperatures. From this plot is clear that an ohmic electrical contact between the gold contact pads and the PEDOT:TsO nanowires was established as the current voltage relation was linear. The proper electrical contact is ensured by the large contact area between the PEDOT:TsO nanowires and the gold electrodes combined with the smooth corner of the electrodes. The major difference, between this and previous fabrication approaches, is that the PEDOT:TsO in this case is first spin coated on the electrodes and patterned at the electrodes yielding the good electrical contact. In Fig. 3b, an image acquired with conductive AFM is included to show the complete removal of the isolating peptide nanotubes from the surface of the PEDOT:TsO.

\subsection{Temperature measurements}

Finally, to demonstrate the potential of the PEDOT:TsO nanowires they were used as temperature sensors. The impedance of the PEDOT:TsO nanowire was monitored as the temperature was changed. In Fig. 4a, the impedance of the wire is plotted as a function of the temperature of the solution in which the PEDOT:TsO nanowires devices wrapped in a thin polymer foil were submerged (determined by external temperature sensing). In this experiment, a linear correlation between the impedance of the PEDOT:TsO nanowires and the external temperature was seen. From this plot it is also evident a low noise level in the PEDOT:TsO nanowire, corresponding to less than $0.05^{\circ} \mathrm{C}$. This is further demonstrated in Fig. $4 \mathrm{~b}$ where the impedance is monitored while the temperature is changed $0.2^{\circ} \mathrm{C}$. As a result of this change in temperature the impedance of the PEDOT:TsO nanowire increased more than 8 times the noise level. Note that in this measurement no 


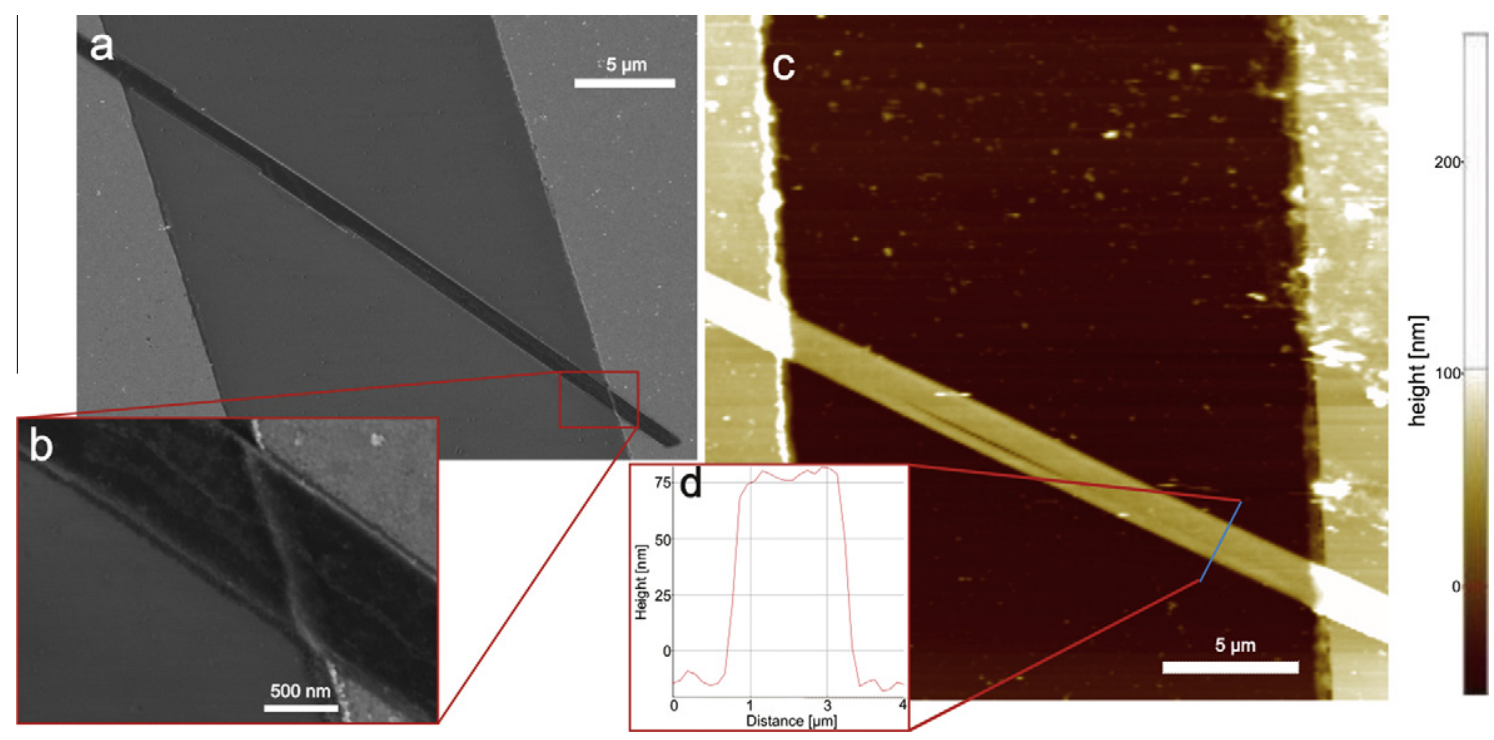

Fig. 2. Visualization of the fabricated PEDOT:TsO nanowire structures with the gold contact pads visible. A SEM image of the whole PEDOT: TsO nanowire spanning the gap between two of the gold electrodes is seen in (a). In (b) a zoom of the contact area between the PEDOT:TsO nanowire and the gold contacts is shown. In this image it is seen that the PEDOT:TsO nanowire covers the step of the gold contact very well, which is also evident in the electrical recordings presented below. In (c) a AFM image of the junction between the PEDOT:TsO nanowire and the gold contact pads is seen. In (d) a line scan covering the PEDOT:TsO wire is included to verify the height of the fabricated PEDOT:TsO nanowire. From this scan the thickness of the nanowire can be estimated to $75 \mathrm{~nm}$.

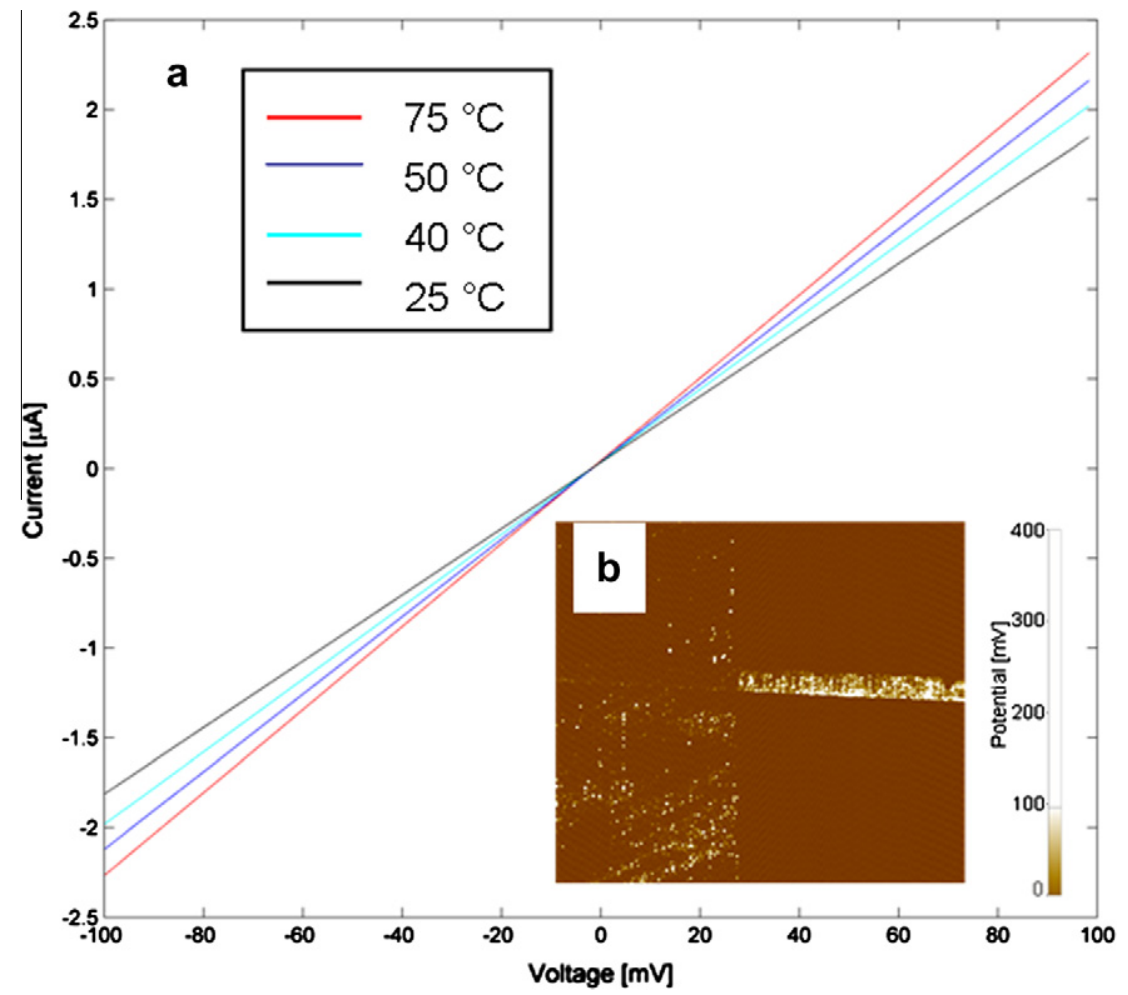

Fig. 3. $I-V$ curves for the nanowire devices at different temperatures is plotted to demonstrate the completely linear ohmic behavior of the PEDOT:TsO nanowire indicating a good contact between the gold electrodes and the PEDOT:TsO nanowire (a). In (b) an image acquired with conductive AFM is included to show the complete removal of the isolating peptide nanotubes from the surface of the PEDOT:TsO.

shielding of the signal was conducted to mimic the situation in real temperature sensing environments. Performing the measurements in a faraday cage would result in an even smaller noise level and hence higher sensitivity. 

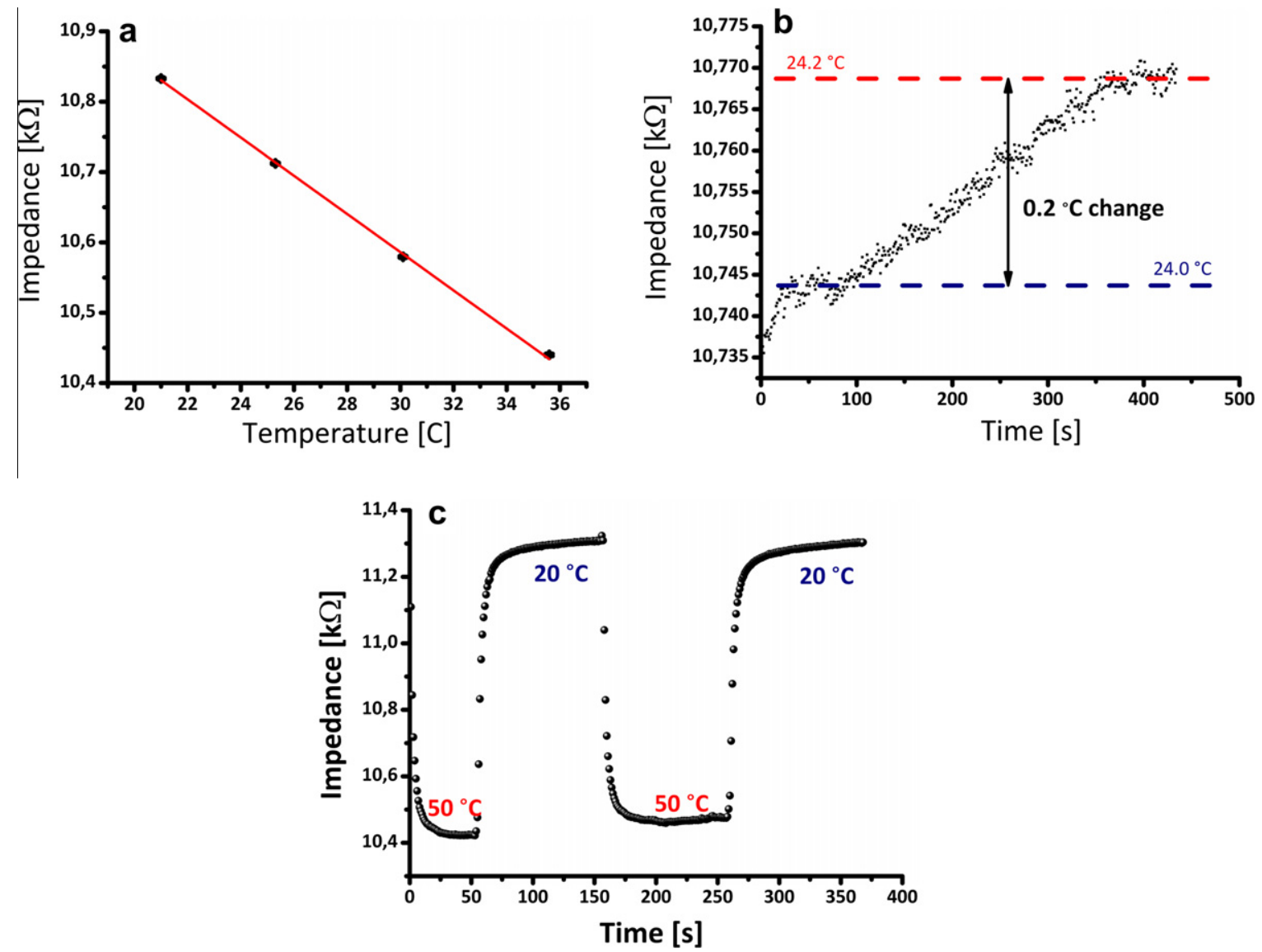

Fig. 4. To demonstrate the potential of these PEDOT:TsO nanowire devices they have been utilized as temperature sensors. In (a), the relationship between temperature and impedance is plotted and from this plot it is seen that the temperature-impedance relation is linear. In (b), the sensitivity of the PEDOT:TsO nanowire device to temperature changes is illustrated. In this case the temperature was changed with $0.2{ }^{\circ} \mathrm{C}$ which resulted in a signal change of more than 8 times the noise floor. To determine the response time of the PEDOT:TsO nanowire devices to changes in external temperatures the impedance of the nanowire was continuously monitored as the device was transferred between two water baths with different temperatures as seen in (c). Within a few seconds the impedance has changed to the new value. Note that the major part of the response time in this setup stems from the thermal mass of the substrate on which the PEDOT:TsO nanowire is fabricated. The response time of the wire itself is much faster.

During the experiments it was also noted that the response time of the PEDOT:TsO nanowire temperature sensor was shorter than that of the traditional external temperature sensor. Therefore in Fig. 4 the response time of the PEDOT:TsO nanowire device is investigated by the repeated change of external temperature of the wire. From this measurement it is seen that within $10 \mathrm{~s}$ the PEDOT:TsO nanowire device has equilibrated to the new temperature. The fast response of the PEDOT:TsO nanowire stem from the small thermal mass of the wire. In the specific experiments, in fact, the major part of the thermal mass and hence the major contribution to the response time stems from thermal mass of the substrate wafer on which the PEDOT:TsO nanowire is fabricated. The PEDOT:TsO nanowire itself has a thickness of $50 \mathrm{~nm}$, a length of $10 \mu \mathrm{m}$ and a width in the order of $500 \mathrm{~nm}$ and hence the volume of the wire is only $0.25 \mu \mathrm{m}^{3}$ compared to the $\sim 10^{17} \mu \mathrm{m}^{3}$ of the substrate. In comparison with other temperature sensors such as strings and bimetallic geometries relying on optical readout [20,28-30], the PEDOT:TsO nanowire only requires a 2 point electrical readout that, due to the small impedance, in principle can be read using a multimeter depending on the desired precision. Temperature measurement with nanowire structures is not a new principle and has previously been demonstrated with both PEDOT nanowires [15] and nanowires of other materials [31-33]. The PEDOT:TsO nanowire fabricated in this work has a much lower resistivity than the previously demonstrated nanowire devices. Therefore the readout procedure for the nanowire devices fabricated in this work is easier and as mentioned above can be conducted with a standard 2 point electrical read out procedure. The main benefit is however the easy fabrication scheme demonstrated in our work allowing the fabrication of nanowire devices in less than half a day.

The very low contact resistance between the gold electrode and the PEDOT:TsO nanowire ensure very sensitive measurements since the dominant change to the impedance of the device is the impedance of the PEDOT:TsO nanowire. Hence, any smaller change in the impedance in the gold contact pads and electrodes due to the temperature change can be disregarded. In order to utilize the 
PEDOT:TsO nanowires as biosensors in liquid conditions one will have to pattern a passivation layer covering the gold electrodes to ensure no parasitic current through the medium in which the sensors are operated.

\section{Conclusions}

In this work, we have combined the benefits of bottom up fabrication using self-assembled peptide structures for the patterning of PEDOT:TsO nanowires with the benefits of top down fabrication of macroscopic gold electrode patterns to provide contact pads for reliable electrical contact. In this way we have demonstrated a rapid and low cost fabrication method for the preparation of PEDOT:TsO nanowire devices (the entire fabrication process can be conducted in approximately $5 \mathrm{~h}$ ). The fabrication procedure of the PEDOT:TsO layer avoided the use of organic solvents due to the easy removal of the peptide nanotubes in water. The fabricated PEDOT:TsO nanowires were used as sensitive sensors to measure temperature changes (down to $0.05{ }^{\circ} \mathrm{C}$ changes can be detected with the current setup) with a fast response time (in this setup approximately $10 \mathrm{~s})$.

\section{References}

[1] Y. Cui, Q.Q. Wei, H.K. Park, C.M. Lieber, Nanowire nanosensors for highly sensitive and selective detection of biological and chemical species, Science 293 (2001) 1289-1292.

[2] C.Y. Hsiao, C.H. Lin, C.H. Hung, C.J. Su, Y.R. Lo, C.C. Lee, H.C. Lin, F.H. Ko, T.Y. Huang, Y.S. Yang, Novel poly-silicon nanowire field effect transistor for biosensing application, Biosens. Bioelectron. 24 (2009) $1223-1229$.

[3] W.E. Svendsen, M. Jorgensen, L. Andresen, K.B. Andersen, M.B.B. Larsen, S. Skov, M. Dimaki, Silicon Nanowire as Virus Sensor in a Total Analysis System, Eurosensors XXV, 2011.

[4] G.J. Zhang, L. Zhang, M.J. Huang, Z.H.H. Luo, G.K.I. Tay, E.J.A. Lim, T.G. Kang, Y. Chen, Silicon nanowire biosensor for highly sensitive and rapid detection of dengue virus, Sens. Actuat. B-Chem. 146 (2010) $138-144$.

[5] G.F. Zheng, F. Patolsky, Y. Cui, W.U. Wang, C.M. Lieber, Multiplexed electrical detection of cancer markers with nanowire sensor arrays, Nat. Biotechnol. 23 (2005) 1294-1301.

[6] K. Balasubramanian, Challenges in the use of 1D nanostructures for on-chip biosensing and diagnostics: a review, Biosens. Bioelectron. 26 (2010) 1195-1204.

[7] I. Lee, X. Luo, J. Huang, X.T. Cui, M. Yun, Detection of cardiac biomarkers using single polyaniline nanowire-based conductometric biosensors, Biosensors 2 (2012) 205-220.

[8] I. Lee, X.L. Luo, X.T. Cui, M. Yun, Highly sensitive single polyaniline nanowire biosensor for the detection of immunoglobulin $G$ and myoglobin, Biosens. Bioelectron. 26 (2011) 3297-3302.

[9] X.L. Luo, I. Lee, J.Y. Huang, M.H. Yun, X.Y.T. Cui, Ultrasensitive protein detection using an aptamer-functionalized single polyaniline nanowire, Chem. Commun. 47 (2011) 6368-6370.

[10] A. Das, C.H. Lei, M. Elliott, J.E. Macdonald, M.L. Turner, Nonlithographic fabrication of PEDOT nano-wires between fixed Au electrodes, Org. Electron. 7 (2006) 181-187.
[11] Y.Z. Long, J.L. Duvail, Z.J. Chen, A.Z. Jin, C.Z. Gu, Electrical conductivity and current-voltage characteristics of individual conducting polymer PEDOT nanowires, Chin. Phys. Lett. 25 (2008) 3474-3477.

[12] Y.Z. Long, J.L. Duvail, Z.J. Chen, A.Z. Jin, C.Z. Gu, Electrical properties of isolated poly(3,4-ethylenedioxythiophene) nanowires prepared by template synthesis, Polym. Adv. Technol. 20 (2009) 541-544.

[13] Y.X. Chen, Y. Luo, Precisely defined heterogeneous conducting polymer nanowire arrays - fabrication and chemical sensing applications, Adv. Mater. 21 (2009) 2040-2044.

[14] C.M. Hangarter, S.C. Hernandez, X.I. He, N. Chartuprayoon, Y.H. Choa, N.V. Myung, Tuning the gas sensing performance of single PEDOT nanowire devices, Analyst 136 (2011) 2350-2358.

[15] Y.Z. Long, J.L. Duvail, M.M. Li, C.Z. Gu, Z.W. Liu, S.P. Ringer, Electrical conductivity studies on individual conjugated polymer nanowires: two-probe and four-probe results, Nanoscale Res. Lett. 5 (2010) 237-242.

[16] J.A. Arter, J.E. Diaz, K.C. Donavan, T. Yuan, R.M. Penner, G.A. Weiss, Virus-polymer hybrid nanowires tailored to detect prostate-specific membrane antigen, Anal. Chem. 84 (2012) 2776-2783.

[17] J.A. Arter, D.K. Taggart, T.M. McIntire, R.M. Penner, G.A. Weiss, VirusPEDOT nanowires for biosensing, Nano Lett. 10 (2010) 4858-4862.

[18] B. Kannan, D.E. Williams, C. Laslau, J. Travas-Sejdic, A highly sensitive, label-free gene sensor based on a single conducting polymer nanowire, Biosens. Bioelectron. 35 (2012) 258-264.

[19] R.H. Farahi, A. Passian, L. Tetard, T. Thundat, Critical issues in sensor science to aid food and water safety, ACS Nano 6 (2012) 4548-4556.

[20] K.B. Andersen, J. Castillo-Leon, T. Bakmand, W.E. Svendsen, Alignment and use of self-assembled peptide nanotubes as dryetching mask, Jpn. J. Appl. Phys. 51 (2012).

[21] M.B. Larsen, K.B. Andersen, W.E. Svendsen, J. Castillo-León, Selfassembled peptide nanotubes as an etching material for the rapid fabrication of silicon wires, BioNanoScience 1 (2011) 31-37.

[22] J. Castillo, S. Tanzi, M. Dimaki, W. Svendsen, Manipulation of selfassembly amyloid peptide nanotubes by dielectrophoresis, Electrophoresis 29 (2008) 5026-5032.

[23] J.J. Castillo, W.E. Svendsen, N. Rozlosnik, P. Escobar, F. Martínez, J. Castillo-León. Detection of cancer cells using a peptide nanotubefolic acid modified graphene electrode, Analyst 138 (2013) 10261031.

[24] J. Castillo-León, R. Rodriguez-Trujillo, S. Gauthier, A.C.O. Jensen, W.E. Svendsen, Micro-"factory" for self-assembled peptide nanostructures, Microelectron. Eng. 88 (2011) 1685-1688.

[25] C.H. Clausen, M. Dimaki, S.P. Panagos, E. Kasotakis, A. Mitraki, W.E. Svendsen, J. Castillo-León, Electrostatic force microscopy of selfassembled peptide structures, Scanning 33 (2011) 201-207.

[26] C.H. Clausen, J. Jensen, J. Castillo, M. Dimaki, W.E. Svendsen, Qualitative mapping of structurally different dipeptide nanotubes, Nano Lett. 8 (2008) 4066-4069.

[27] K.B. Andersen, J. Castillo-León, M. Hedstrom, W.E. Svendsen, Stability of diphenylalanine peptide nanotubes in solution, Nanoscale 3 (2011) 994-998.

[28] H. Kang, D. Kim, M. Song, Sensitivity enhancement of FBG temperature sensor, Optical Sensing and Detection Ii, 2012.

[29] T. Larsen, S. Schmid, L. Gronberg, A.O. Niskanen, J. Hassel, S. Dohn, A. Boisen, Ultrasensitive string-based temperature sensors, Appl. Phys. Lett. 98 (2011)

[30] A.K. Pandey, O. Gottlieb, O. Shtempluck, E. Buks, Performance of an AuPd micromechanical resonator as a temperature sensor, Appl. Phys. Lett. 96 (2010).

[31] A. Menzel, K. Subannajui, F. Guder, D. Moser, O. Paul, M. Zacharias, Multifunctional ZnO-nanowire-based sensor, Adv. Funct. Mater. 21 (2011) 4342-4348.

[32] C.P. Wang, C.W. Liu, C. Gau, Silicon nanowire temperature sensor and its characteristic, in: NEMS 2011 - Sixth IEEE International Conference on Nano/Micro Engineered and Molecular Systems, 2011, pp. 630-633.

[33] A.M. Zaitsev, A.M. Levine, S.H. Zaidi, Carbon nanowire-based temperature sensor, Phys. Status Solidi A 204 (2007) 3574-3579. 